\title{
Reduction of vibrations in mechanical systems using piezoelectric elements
}

\author{
Katarzyna Bialas* \\ Silesian University of Technology University, Faculty of Mechanical Engineering, Institute of \\ Engineering Processes Automation and Integrated Manufacturing Systems, Konarskiego 18A, 44-100 \\ Gliwice, Poland
}

\begin{abstract}
The aim of study is to visualize not only the primary and active element but also sensors, returning the current state of the system. The work shows the use of piezoelectric accelerometer sensors in the active vibration reduction. In addition to the elements reduction of vibrations also are necessary elements with which it will be possible to constantly test and measure vibration. It is necessary to generate the force to appropriate executive to reduce the effects of vibration. It will be also shows how to design basic system in something special way which is the synthesis of mechanical systems.
\end{abstract}

\section{Introduction}

Mechanical vibrations are one of the frequent phenomena occurring in everyday life. Most vibrations have a detrimental effect on the environment in which it occurs. The vibration phenomenon is often a side effect of machines and devices. These actions have an adverse effect on the equipment, causing their faster wear and causing their incorrect operation. The goal of constructors of new machines and devices is to achieve lower operating costs and increase productivity.

The structure of modern devices is more complex compared to older models. Vibrations also have a negative effect on the human body, especially vibrations with a low frequency [1-3]. The aim of this study is to present the sensors transmitting the current state of the system as well as the basic system and the active element reducing vibrations. The paper presents the use of piezoelectric accelerometers as vibration sensors in active vibration reduction. The system presented in this work can be designed as a basic system with an additional active subsystem. Non-classical methods of designing systems with active elements reduction of vibrations were presented in earlier studies [4,5]. It is also possible to design an active subsystem for an existing basic system. An additional subsystem reduces unwanted vibrations. The active subsystem can be implemented differently. In this example shown, it is implemented using electrical components.

The presented systems require elements with the use of which it will be possible to continuously test and measure vibrations. The use of active elements is designed to

\footnotetext{
*Corresponding author: katarzyna.bialas@polsl.pl
} 
eliminate unwanted vibrations. The sensors control the current state of the system, and enables generation of adequate force by the active element [6-9].

\section{Piezoelectric accelerometer}

Vibration sensors are devices that process the value of displacements, velocities or accelerations into an electrical signal. Vibration measurement can be carried out in two different ways. The recording of vibrations relative to the fixed reference system is called absolute measurement. Relative measurement consists in incorporating an additional element into the system and measuring its vibrations in relation to the tested object. In sensors, this element is called the seismic mass [10].

For vibration measurements in the absolute methods most commonly used non-contact proximity sensors [10]:

- electromagnetic,

- induction,

- eddy current,

- capacitive.

However, when measuring using relative methods, accelerometers are the most popular [10]:

- piezoelectric,

- electrodynamic.

The operation of piezoelectric accelerometers is based on the phenomenon of electric charge generation on the walls of piezoelectric materials under the influence of deforming forces. During vibrating motion, mechanical forces due to the inertia of the seismic mass act on the piezoelectric element. As a result, an electric charge is generated. Piezoelectric materials generate a charge proportional to the value of the force that affects them [10-12].

$$
Q_{s}=d F_{c}
$$

Where:

$Q_{s}$ - charge generated by piezoelectric elements

$d$ - piezoelectric coefficient

$F_{c}-$ force on the piezoelectric element

At a constant seismic mass, the force exerted on the piezoelectric element is proportional to the acceleration value. In the case of usable frequencies of the sensor, we obtain a simple proportional dependence of the generated charge (voltage) on acceleration. This dependence is referred to as the sensitivity of the sensor.

Charge sensitivity

$$
S_{q}=\frac{q}{a}
$$

Voltage sensitivity

$$
S_{u}=\frac{u}{a}
$$

Where:

$q$ - charge

$u$ - voltage

$a$ - accelerate

The Figure 1 is shown model concept than simulations the action of a piezoelectric sensor in the Matlab program. 


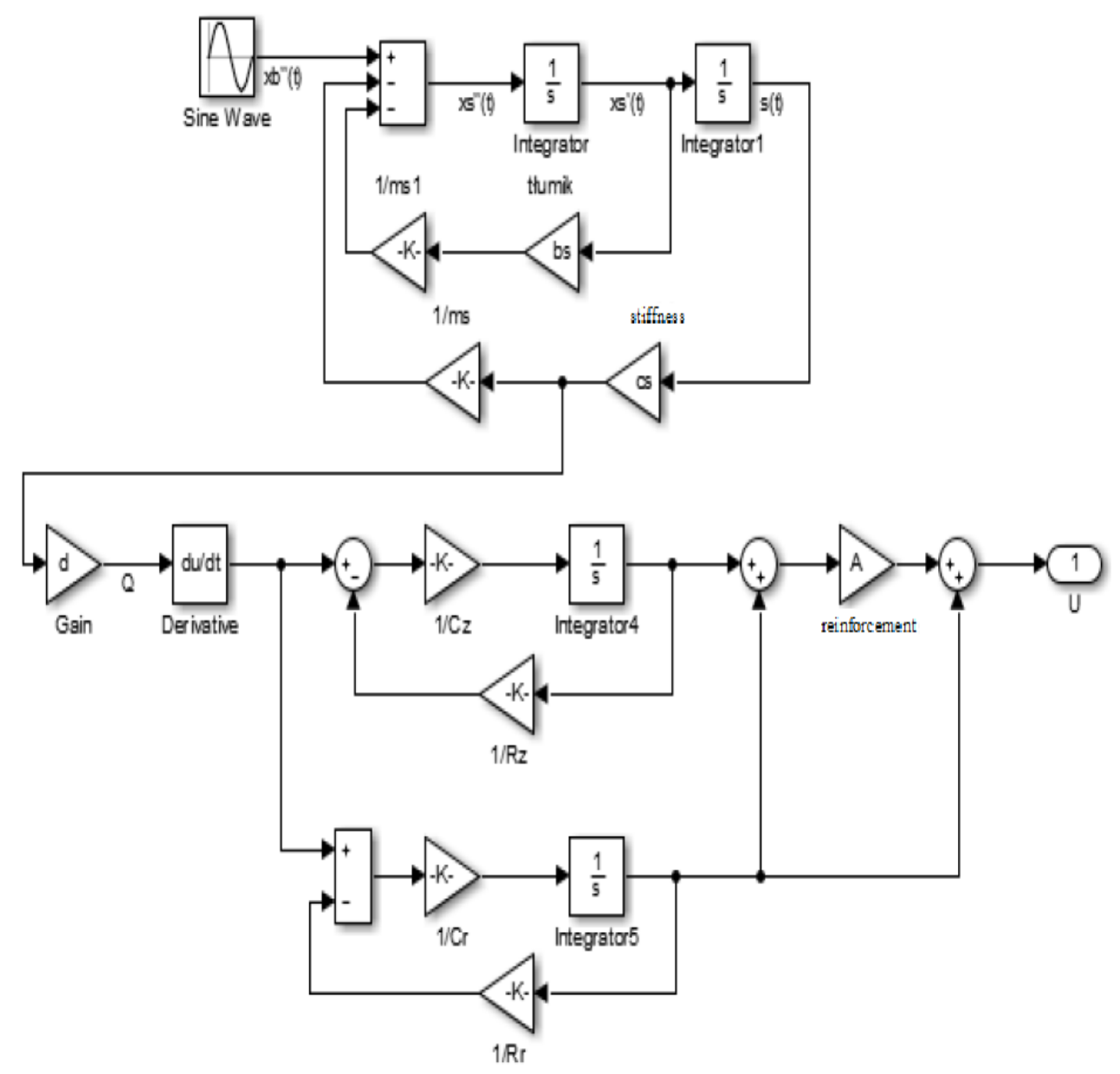

Fig. 1. A program that simulates the action of an piezoelectric sensor.

\section{Research problem and method}

The system under consideration Figure 2 consists of two inertial elements and two elastic elements. $\boldsymbol{F}(\mathrm{F}=5 \sin \omega \mathrm{t}[\mathrm{kN}])$ force is at the first inertial element.

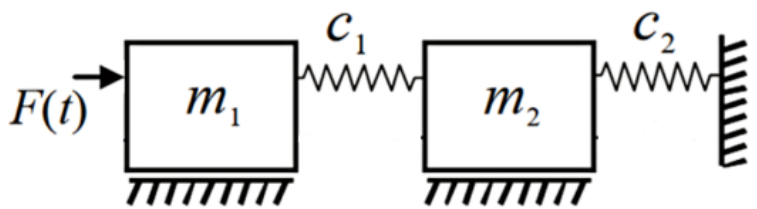

Fig. 2. The system under consideration. 


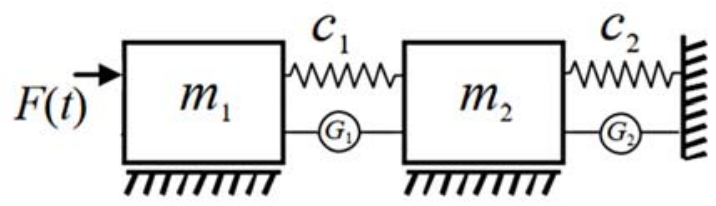

Fig. 3. The system with active elements.

Table 1. The values of passive elements

\begin{tabular}{|l|c|c|}
\hline No. & element & value \\
\hline 1. & $\mathrm{~m}_{1}$ & $5[\mathrm{~kg}]$ \\
\hline 2. & $\mathrm{~m}_{2}$ & $7[\mathrm{~kg}]$ \\
\hline 3. & $\mathrm{c}_{1}$ & $200[\mathrm{~N} / \mathrm{m}]$ \\
\hline 4. & $\mathrm{c}_{2}$ & $300[\mathrm{~N} / \mathrm{m}]$ \\
\hline
\end{tabular}

The work is limited to using only active elements Figure 3. In the case of passive components, viscose dampers could be used.

In order to determine values of forces $G_{1}$ and $G_{2}$ generated by active elements, it is necessary to solve the system of equations (3), in the matrix form [6-8].

$$
G=D \cdot A-F
$$

Where:

$G$ - matrix of excitations generated by active elements,

$D$ - matrix of dynamic stiffness,

$A$ - matrix of amplitudes (approaching zero),

$F$ - matrix of dynamic excitations.

Table 2. The values of active elements

\begin{tabular}{|l|l|l|}
\hline No. & \multirow{2}{*}{ Frequency } & $\begin{array}{c}\text { The value of amplitude of force } \\
\text { generated by active element }[\mathrm{k} N]\end{array}$ \\
\hline 1. & \multirow{2}{*}{$\omega=4[\mathrm{rad} / \mathrm{s}]$} & $G_{1}=14,7$ \\
& & $G_{2}=0,52$ \\
\hline 2. & \multirow{2}{*}{$\omega=10[\mathrm{rad} / \mathrm{s}]$} & $G_{1}=15,7$ \\
\cline { 3 - 3 } & & $G_{2}=1,9$ \\
\hline 4. & &
\end{tabular}

An analysis of the system concerned. Diagrams of amplitude-frequency characteristics of the system without damping and a system with active vibration reduction were drawn up Figure 4 .

\section{Conclusions}

In the case of systems without reduction, clear peaks can be seen in the graphs of amplitude-frequency characteristics. A sudden increase in the amplitude value appears at frequencies corresponding to resonance frequencies. In systems with active reduction of vibration, the value of amplitudes close to zero in the whole range of the frequencies tested was obtained. The considerations presented in the paper are an introduction to the problem of using sensors in active vibration reduction. In subsequent studies, the use of other sensors should be checked. Investigate the interaction of particular elements (basic system, 
active elements, sensors). After simulation, you can build a prototype and also, we can compare it with the simulation results.

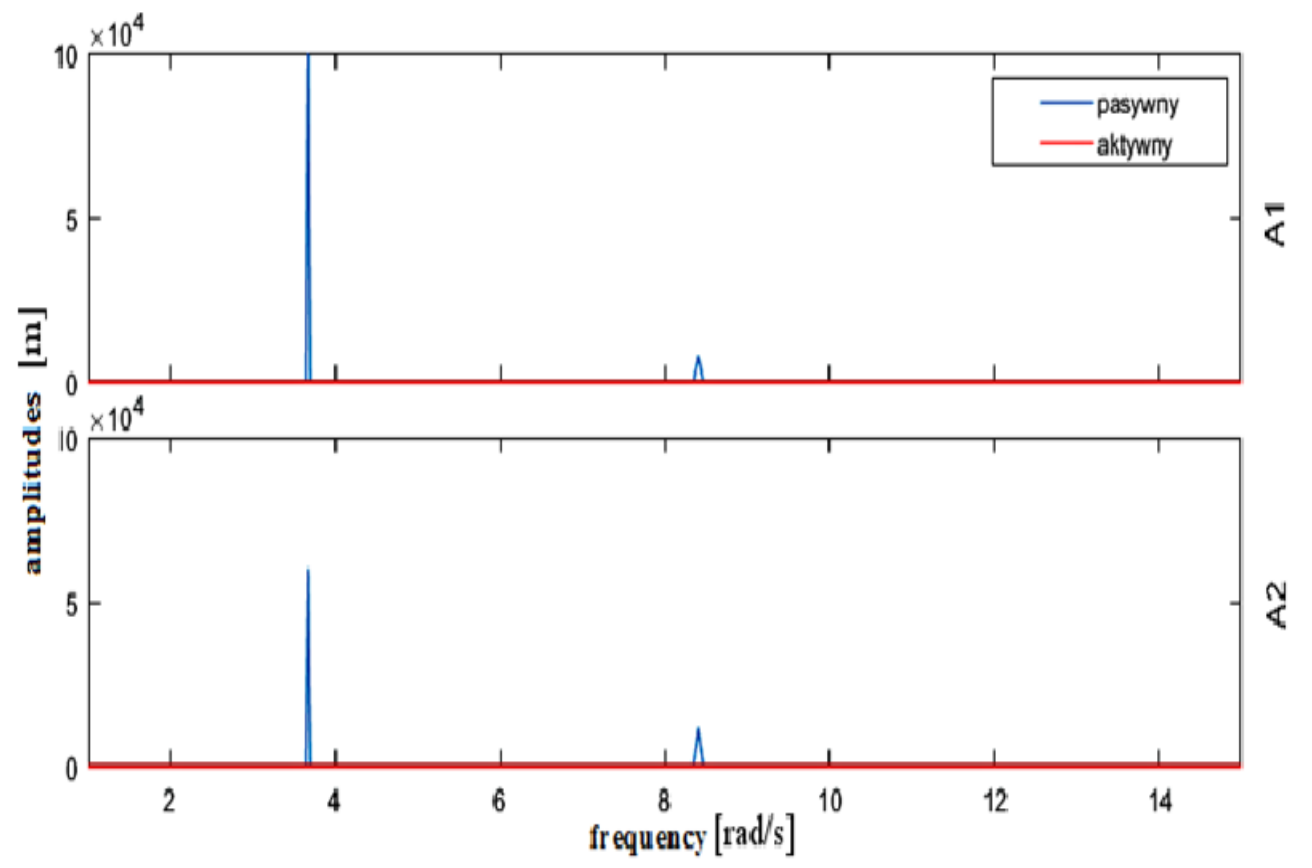

Fig. 4. Diagrams of amplitude-frequency characteristics.

\section{References}

1. Z. Engel, J. Kowal, Kraków (in Polish), AGH Press, (1995)

2. T. Kucharski, Wydawnictwo Naukowo-Techniczne, Warszawa, (in Polish), (2002)

3. S. Michałowski, (in Polish). 171, 1994)

4. K.Białas, A.Buchacz IOP Conf. Series-Materials Science and Eng. 95012051 (2015)

5. K.Białas, A.Buchacz, T.Dzitkowski, Gliwice Monograph 230, Silesian Univ. of Techn. Press, (in Polish). (2009)

6. K. Bialas Applied Mechanics and Materials; 371, 657-661 (2013)

7. K. Białas Int. Conf. ModTech Proceedings I, II, (2012)

8. K. Białas, A. Buchacz The Influence of Changing the Parameters of Electrical Components Implementing the Active Reduction of Vibration App. Mech. and Materials, 657, 614-618 (2014)

9. A.Buchacz, M.Płaczek Int. J. of Modern Manuf. Tech, II, 1 (2010)

10. M. Serridge, T. R. Licht Piezoelectric accelerometer and vibration preamplifier handbook, Brüel \& Kjaer, Glostrup (1987)

11. G. C. Onwubolu Mechatronics Principles and Applications, Elsevier ButterworthHeinemann (2005)

12. A. Preumont MecH Dynamics, Springer. The Netherlands (2006) 